Original Paper http://ajol.info/index.php/ijbcs http://indexmedicus.afro.who.int

\title{
Impacts socio-économiques sur les cuniculteurs de la vaccination des lapins contre les coccidioses intestinales au Bénin
}

\author{
Y. AKPO ${ }^{1 *}$, M. T. KPODEKON ${ }^{2}$, Y. DJAGO ${ }^{2}$ et D. LICOIS $^{3}$ \\ ${ }^{I}$ Faculté d'Agronomie de l'Université de Parakou, Laboratoire d'Ecologie, de Santé et de Productions \\ Animales (LESPA), BP 123 Parakou, Bénin. \\ ${ }^{2}$ Ecole Polytechnique d'Abomey-Calavi - Laboratoire de Recherche en Biologie Appliquée (LARBA), \\ Unité de Recherche Cunicole et Cavicole (URCC), 01 BP 2009 Cotonou, Bénin. \\ ${ }^{3}$ INRA - Tours, Laboratoire de Pathologie du Lapin, BP 37380 Nouzilly, France. \\ *Auteur correspondant ; E-mail : yao.akpo@gmail.com; Tél : (+229) 21146807 ;
}

\section{REMERCIEMENTS}

Nous adressons nos sincères remerciements aux Programmes CORUS, NPT/BEN/146, au CODESRIA et au MESRS pour leurs soutiens techniques et financiers.

\section{RESUME}

Les coccidioses constituent l'une des maladies les plus meurtrières chez les lapins. Pour mieux contrôler cette affection, une prophylaxie vaccinale est mise au point. La réussite d'une telle innovation passe par la perception des cuniculteurs. Ainsi, la présente étude a été entreprise afin de déterminer les facteurs favorisant l'adoption par les éleveurs de la vaccination des lapins contre les coccidioses. Les enquêtes menées auprès des cuniculteurs ont révélé que $100 \%$ d'entre eux ont une opinion favorable du vaccin. De même, 83,3\% des éleveurs ont affirmé que le vaccin était efficace. Par ailleurs, $80 \%$ des éleveurs ont déclaré connaître la coccidiose. Parmi ceux-ci, 62,5\% avait bien décrit les signes cliniques de la coccidiose. L'étude économique a montré que la vaccination engendre un bénéfice net de 123,2 F CFA par lapin comparativement aux lapins non vaccinés. La vaccination s'avère efficace et est bien appréciée par les utilisateurs. Son adoption contribuera à améliorer les conditions de vie des cuniculteurs.

(C) 2015 International Formulae Group. All rights reserved.

Mots clés : Oryctolagus cuniculus, cuniculture, coccidies, prophylaxie, immunisation, adoption.

\section{Socio-economic effects on the rabbit breeders of rabbit's vaccination against intestinal coccidiosis in Benin}

\section{ABSTRACT}

Coccidiosis is recognized as being one of the major serious diseases in rabbit breeding. For a better control of this disease, a vaccination program is set up. The perception of the rabbit breeders determines the success of this innovation. Thus, this study was undertaken in order to determine the factors which favour the adoption of the rabbit vaccination against coccidiosis by the rabbit breeders. Survey performed on rabbit 
breeding revealed that $100 \%$ of the breeders included in the survey thought that vaccine is useful for the control of coccidiosis. An average of $83.3 \%$ of breeders has reported that the use of vaccine was effective to control coccidiosis. Likewise among rabbit breeders included in the study, an average of $80 \%$ has been reported to know coccidiosis. Among those breeders, an average of $62.6 \%$ was reported to know well clinical symptoms of coccidiosis. Economical study carried out on rabbit breeding showed that vaccine involves a net profit of 132.2 F CFA per vaccinated rabbit compared to non vaccinated rabbits. Vaccine turns out to an effective control of coccidiosis in rabbit and was well appreciated by the breeders. Its adoption will help improving rabbit breeders' life.

(c) 2015 International Formulae Group. All rights reserved.

Keywords: Oryctolagus cuniculus, rabbit breeding, coccidia, prevention, immunization, adoption.

\section{INTRODUCTION}

La chair du lapin constitue l'une des viandes les plus recherchées au Bénin, parce qu'elle est tendre, savoureuse, peu grasse et nutritive avec un faible taux de cholestérol (Kpodékon et al., 2000). En conséquence, la demande sur le marché national est aujourd'hui en forte augmentation. Ceci a suscité auprès des populations béninoises, une vocation à l'élevage du lapin. Ainsi, environ $60 \%$ des Béninois entreprendraient la cuniculture si l'opportunité leur en était offerte. Le Bénin peut donc faire de la spéculation lapin de chair, un des moteurs de croissance économique, de sécurité alimentaire, de lutte contre la pauvreté et le chômage. En effet, depuis une vingtaine d'années, l'élevage du lapin connaît un grand essor au Bénin grâce à la mise en place en 1987 du Centre Cunicole de Recherche et d'Information (CECURI) à l'Ecole Polytechnique d'Abomey-Calavi et de l'Association Béninoise des Cuniculteurs $(\mathrm{ABeC})$ en 1993. La production béninoise de viande de lapin est estimée à 1000 tonnes par an et fait vivre 9000 foyers environ, professionnels et non professionnels $(\mathrm{ABeC}$, 2008).

Malgré son importance microéconomique aussi bien dans les villages que dans les zones péri-urbaines (Kpodékon et al., 2000; Djago et al., 2010), le développement de la cuniculture reste handicapé par plusieurs contraintes parmi lesquelles les facteurs d'ordre pathologique.

Les coccidioses sont reconnues comme une dominante pathologique parmi les parasitoses du lapin. Elles sont les plus dangereuses chez cette espèce animale et constituent un problème sanitaire majeur de l'élevage cunicole partout où cet élevage est pratiqué (Akpo et al., 2011). Cette maladie entraîne des pertes économiques considérables, en raison de la baisse de productivité et des mortalités qu'elle induit (Colombo et Zago, 2003).

Les travaux menés au Bénin par Akpo et al. (2012) confirment l'omniprésence des coccidies dans la majorité des élevages cunicoles. Les espèces de coccidies les plus fréquemment rencontrées sont $E$. magna et $E$ media (Djago et al., 2010). Les maladies provoquées par ces coccidies frappent surtout les lapereaux après le sevrage (Colombo et Zago, 2003). Les mesures de prévention contre cette maladie doivent être prises avant le sevrage. Pendant longtemps, la lutte contre les coccidioses du lapin a reposé sur l'administration d'anticoccidiens en continu dans l'aliment ou dans l'eau de boisson. Outre leur coût élevé, ces médicaments ont fait apparaître dans les élevages des coccidies chimiorésistantes, mais aussi une réticence des consommateurs (Akpo et al., 2012).

La vaccination contre les coccidies pourrait être une alternative à la lutte contre les coccidioses dans les élevages intensifs et semi-intensifs et apparaît comme une solution d'avenir (Pakandl et Jelinkova, 2006, Xu et al., 2008, Shivaramaiah et al., 2014). La réussite d'une telle vaccination à l'échelle de l'élevage du lapin au Bénin, exige une adhésion des cuniculteurs.

L'objectif de ce travail était de 
déterminer les facteurs favorisant l'adoption par les éleveurs de la vaccination des lapins contre les coccidioses.

\section{MATERIEL ET METHODES}

\section{Zone d'étude}

Cette étude s'est déroulée dans 5 départements administratifs du Sud-Bénin, des zones de forte concentration des élevages de lapins à savoir les départements de l'Atlantique, de l'Ouémé, du Plateau, du Mono et du Couffo (Figure 1). Cette zone est située entre $6^{\circ} 16^{\prime}$ et $7^{\circ} 30^{\prime}$ de latitude Nord puis $1^{\circ} 29^{\prime}$ et $2^{\circ} 45$ de longitude Est. Elle jouit d'un climat subéquatorial avec quatre saisons, une grande saison des pluies d'avril à juillet, une petite saison sèche d'août à septembre, une petite saison pluvieuse d'octobre à novembre et une grande saison sèche de décembre à mars. La hauteur pluviométrique varie entre $633 \mathrm{~mm}$ et $1270 \mathrm{~mm}$ avec une moyenne annuelle de $950 \mathrm{~mm}$. Les températures moyennes annuelles enregistrées sont comprises entre $25^{\circ} \mathrm{C}$ et $28{ }^{\circ} \mathrm{C}$ avec des humidités relatives allant de 40 à $97 \%$. Les principaux groupes ethniques sont les Fon et les Yoruba. Les religions dominantes sont l'Animisme, le Christianisme et l'Islam (Adam, 2009).

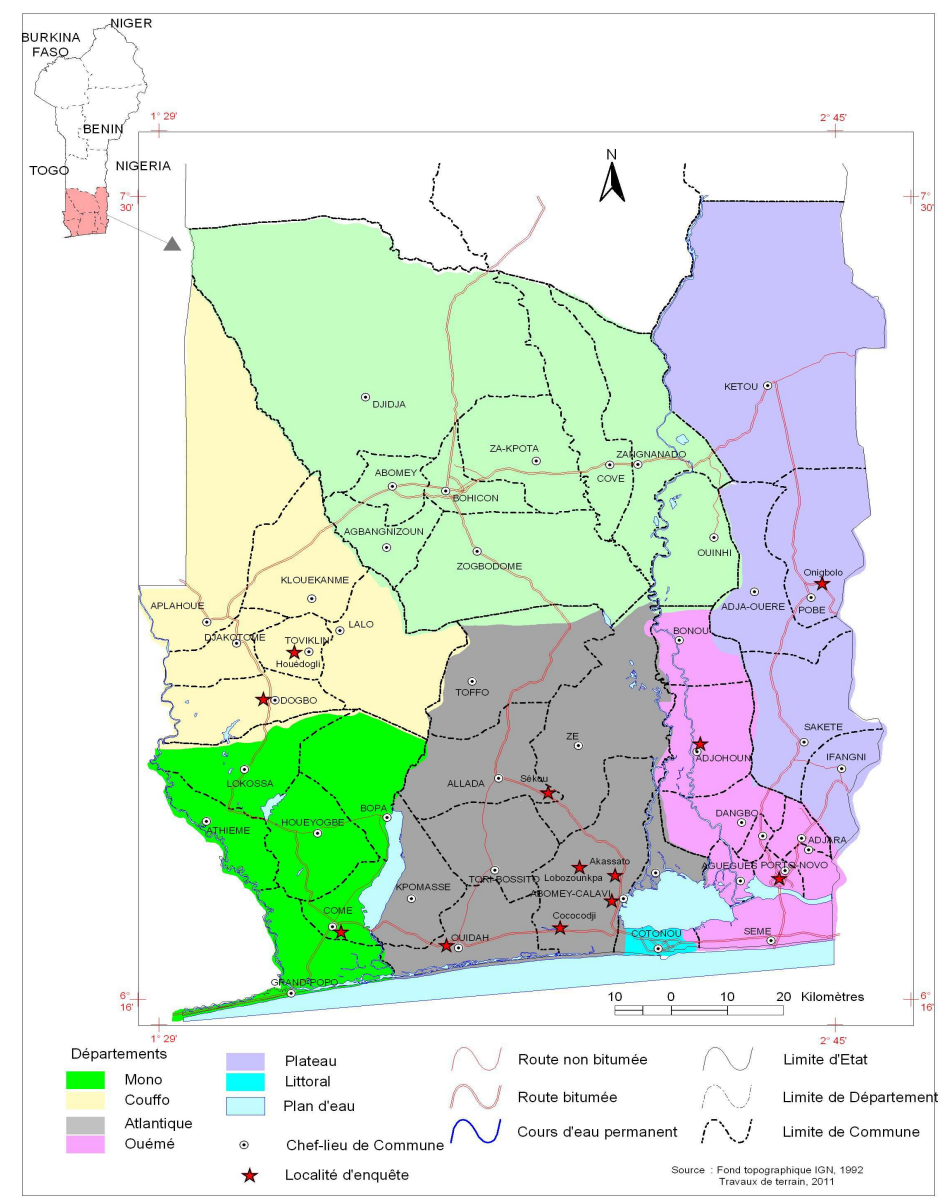

Figure 1: Localisation des élevages cunicoles prospectés. 


\section{Echantillonnage}

Il a été procédé à un échantillonnage raisonné des élevages. Les élevages retenus avaient au moins trois années d'existence et possédaient au moins 20 lapines mères.

\section{Enquête}

Un questionnaire a été élaboré et adressé aux éleveurs de lapins. Les principales rubriques du questionnaire étaient les suivantes: les objectifs de production du cuniculteur, son expérience en matière de cuniculture, l'utilisation antérieure de vaccin, son avis sur le vaccin contre la coccidiose et le prix auquel il était prêt à acheter une dose de vaccin. L'enquête a été menée auprès des six éleveurs de lapins chez qui des vaccinations ont été faites.

\section{Evaluation économique}

Les principaux paramètres retenus étaient le gain moyen quotidien, la quantité d'aliment consommée, le prix de vente et le taux de mortalité des lapins.

\section{Analyse statistique}

Le test bilatéral de $\mathrm{Z}$ du logiciel SAS (Statistical Analysis System, 2010) était utilisé pour la comparaison des différents pourcentages. Un seuil de significativité de $5 \%$ a été fixé.

\section{RESULTATS}

\section{Perception des cuniculteurs sur le vaccin}

Les résultats des enquêtes sont consignés dans le Tableau 1.

Il ressort de ce tableau que $100 \%$ des éleveurs de lapins enquêtés étaient favorables à l'introduction du vaccin. Cette fréquence n'était pas différente $(\mathrm{P}>0,05)$ de celle de $83,3 \%$ des cuniculteurs qui avaient estimé que le vaccin était efficace. Toutefois, cette dernière fréquence a été plus élevée $(\mathrm{P}<$
$0,05)$ que celle de $33,3 \%$ des cuniculteurs qui avaient utilisé dans le passé un vaccin dans leur exploitation. De même, 2 cuniculteurs avaient évoqué les risques liés au caractère vivant $\mathrm{du}$ vaccin. Enfin, en matière d'encadrement, 33,3\% des cuniculteurs étaient suivis par des agents techniques d'élevages. Outre ces données, le prix auquel les éleveurs de lapins étaient prêts à payer pour une dose de vaccin s'élevait à $100 \mathrm{~F} \mathrm{CFA.}$

Outre ces informations, l'enquête avait pris en compte le niveau de connaissance des éleveurs de lapins en matière de coccidioses et les méthodes endogènes utilisées par les cuniculteurs dans la lutte contre les coccidioses. Les résultats consignés dans le Tableau 2 présentent les fréquences des différents paramètres étudiés. Ainsi, $80 \%$ des cuniculteurs avaient déclaré connaître la coccidiose du lapin. Aucune différence significative n'a été observée $(\mathrm{P}>0,05)$ entre cette fréquence et celle de $62,5 \%$ des éleveurs qui avaient décrit correctement les manifestations cliniques de la maladie. Par ailleurs, ces deux fréquences étaient plus élevées $(\mathrm{P}<0,05)$ que celles de $12,5 \%$ et $18,75 \%$ des cuniculteurs qui avaient respectivement recours uniquement à l'utilisation de plantes médicinales et à l'association de médicaments vétérinaires et de plantes médicinales. Les principales plantes médicinales utilisées ont été : Sarcocephalus latifolius, Azadirachta indica et Vernonia amygdalina.

\section{Etude économique}

Les coûts et bénéfices bruts réalisés avec chaque lot de lapins, ont été évalués par rapport à l'aliment, au taux de mortalité et au gain de poids (Tableau 3). Avec un indice de consommation égal à 4 , le bénéfice brut correspondant était de 123,2 FCFA par lapin en faveur du lot vacciné. 
Tableau 1: Résultats des enquêtes auprès des cuniculteurs sur la perception du vaccin.

\begin{tabular}{lc}
\hline Paramètres & Fréquence (\%) \\
\hline Opinions favorables vis-à-vis du vaccin & $100 \mathrm{a}$ \\
Efficacité du vaccin & $83,3 \mathrm{a}$ \\
Risques liés à l'adoption du vaccin & $33,3 \mathrm{~b}$ \\
Cuniculteurs ayant utilisé une fois un vaccin & $33,3 \mathrm{~b}$ \\
Cuniculteurs suivis par un technicien d'élevage & $33,3 \mathrm{~b}$ \\
Effets secondaires liés à l'administration du vaccin & $16,66 \mathrm{c}$ \\
\hline \multicolumn{1}{c}{ a, b, c : les pourcentages suivis de lettres différentes, diffèrent significativement au seuil de 5\%. }
\end{tabular}

Tableau 2: Résultats des enquêtes auprès des éleveurs sur le niveau de connaissance et les méthodes endogènes de lutte contre les coccidioses.

\begin{tabular}{lc}
\hline Paramètres étudiés & Fréquence (\%) \\
\hline Connaissance de la coccidiose & $80 \mathrm{a}^{*}$ \\
Cuniculteurs utilisant uniquement les médicaments vétérinaires & $68,75 \mathrm{a}^{* *}$ \\
Connaissance des signes cliniques de la coccidiose & $62,5 \mathrm{a}^{* *}$ \\
Eleveurs associant les plantes aux médicaments vétérinaires & $18,75 \mathrm{~b}^{* *}$ \\
Utilisateurs de plantes uniquement & $12,5 \mathrm{~b} * *$ \\
\hline *: Fréquence déterminée par rapport à 20 éleveurs, **: Fréquence déterminée par rapport à 16 éleveurs. a, b : les \\
pourcentages suivis de lettres différentes, different significativement au seuil de 5\%.
\end{tabular}

Tableau 3: Coûts et bénéfices bruts après vaccination des lapins.

\begin{tabular}{lcc}
\hline Désignation & Lot & Résultats \\
\hline Prix d'un kg de lapin (FCFA) & Vacciné & 1500 \\
& Témoin & 1500 \\
Gain de poids vif par lapin (kg) & Vacciné & 1,162 \\
& Témoin & 1,108 \\
Prix de vente du lapin par rapport au gain de poids vif (FCFA) & Vacciné & 1743 \\
& Témoin & 1662 \\
Quantité moyenne d'aliment consommé par lapin (kg) & Vacciné & 4,648 \\
& Témoin & 4,432 \\
Prix du kg d'aliment (FCFA) & Vacciné & 175 \\
& Témoin & 175 \\
Prix de la quantité moyenne d'aliment consommé par lapin (FCFA) & Vacciné & 813,4 \\
& Témoin & 775,6 \\
Bénéfice réalisé par rapport au gain de poids & Vacciné & 929,6 \\
& Témoin & 886,4 \\
Nombre de lapins morts (\%) & Vacciné & 4 \\
& Témoin & 16 \\
Coûts des pertes liées aux mortalités (FCFA) par lapin & Vacciné & 60 \\
Coût de la vaccination par lapin (FCFA) & Témoin & 240 \\
& Vacciné & 100 \\
Bénéfice/perte réalisé par lapin par rapport aux mortalités (FCFA) & Témoin & - \\
Bénéfice global réalisé par lapin vacciné par rapport au lapin non vacciné & Vacciné & 769,6 \\
(FCFA) & Témoin & 646,4 \\
& & 123,2 \\
\hline
\end{tabular}




\section{DISCUSSION}

La totalité des cuniculteurs enquêtés a une bonne opinion sur le vaccin utilisé. Par ailleurs, un éleveur a évoqué des effets secondaires liés à la vaccination. En effet, selon ce dernier, l'administration du vaccin est probablement à l'origine de mortalités dans son élevage. Cette affirmation est semblable à celle rapportée par Ahola et al. (2014) qui indiquent que le public en général, a tendance à surestimer le nombre de mortalités imputables à des risques rares, et à l'inverse à sous-estimer ceux dus aux risques plus courants. Pourtant, la majorité des éleveurs affirme que le vaccin est efficace avec un faible taux de mortalité. La plupart des cuniculteurs n'ont jamais utilisé de vaccin dans leurs exploitations, ce qui traduit leurs faibles expériences en matière de vaccination des lapins. De même, ces éleveurs de lapins ne sont généralement pas suivis par un technicien d'élevage qualifié, ce qui pose un problème d'encadrement technique. De façon générale, la vaccination contre les coccidioses du lapin fait l'objet de différentes appréciations de la part des éleveurs. Cette observation rejoint celle faite par Andersen et Mostue (2011) qui rapportent que la perception des utilisateurs vis-à-vis d'un produit varie selon que le risque lié à celui-ci est connu ou pas mais aussi suivant l'expérience personnelle et le niveau d'instruction.

Les travaux sur le niveau de connaissance des cuniculteurs et des méthodes endogènes de lutte contre les coccidioses révèlent que près des $2 / 3$ des éleveurs de lapins enquêtés connaissent bien la coccidiose et ses signes cliniques. Ceci traduit la forte prévalence de cette maladie en élevage de lapins. Cette affection constitue l'une des dominantes pathologiques en cuniculture au Bénin. Les mêmes observations ont été déjà faites au Bénin par Kpodékon et al. (2000) puis par Akpo et al. (2011). Ce qui confirme l'urgence de trouver une solution définitive et durable à ce fléau constituant une contrainte principale à la promotion de la cuniculture au Bénin.
La décoction et les extraits de nombreuses plantes médicinales sont utilisés par les cuniculteurs pour prévenir ou traiter la coccidiose. Les parties des plantes médicinales que les éleveurs de lapins déclarent utiliser sont les parties amères. C'est ainsi que les feuilles de Vernonia amygdalina et de Azadirachta indica sont utilisées tandis qu'avec Sarcocephalus latifolius, les cuniculteurs ont recours aux racines.

Outre ces pratiques, de nombreux éleveurs de lapins utilisent uniquement les médicaments vétérinaires dont les principaux sont le Trisulmix ${ }^{\circledR}$, le Superhypracox ${ }^{\circledR}$ et l'Anticox ${ }^{\circledR}$.

L'étude économique montre que la vaccination contribue à accroître sensiblement le revenu chez l'éleveur qui la pratique. Ainsi, l'adoption de cette technologie par les cuniculteurs doit leur permettre de générer davantage de ressources nécessaires pour l'amélioration de leurs conditions de vie.

\section{Conclusion}

Sur le plan socio-économique, les cuniculteurs ont une bonne perception sur le vaccin utilisé et la vaccination permet de réaliser un bénéfice net de 123,2 FCFA par lapin. Ainsi, la diffusion de cette technologie pourra améliorer la rentabilité des élevages cunicoles et par conséquent les conditions de vie des cuniculteurs.

L'efficacité de la vaccination se traduit aussi par une marge bénéficiaire en faveur des lapins vaccinés. De plus, tous les éleveurs ayant utilisé le vaccin en ont une bonne opinion.

\section{CONFLITS D'INTERETS}

Les auteurs ne déclarent aucun conflit d'intérêt.

\section{CONTRIBUTIONS DES AUTEURS}

YA est l'auteur principal. Il a conduit les travaux sur le terrain et participé à la rédaction du document; MTK et YD ont pris part à la phase d'enquête et à la rédaction du document; DL a participé à la rédaction du volet lié à la vaccination. 


\section{REFERENCES}

ABeC. 2008. Rapport d'activités du Conseil National de l'Association Béninoise des Cuniculteurs, Cotonou, p. 15.

Adam KS. 2009. Le Bénin en Afrique et dans le monde: Données géographiques, historiques, économiques et socioculturelles. In Langues et Politiques de Langues au Bénin. Ablodé (ed). Laboratoire Ablodé/UAC : Cotonou ; 13-29.

Ahola M, Murto P, Kujala P, Pitkänen J. 2014. Perceiving safety in passenger ships-User studies in an authentic environment. Saf. Sci., 70(2014): 222232. DOI : http://dx.doi.org/10.1016/ j.ssci.2014.05.017

Akpo Y, Kpodékon MT, Djago Y, Licois D, Youssao IAK. 2011. Evaluation de l'innocuité de Eimeria magna et de Eimeria media issues du Bénin en vue de leur utilisation comme souches vaccinales. Int. J. Biol. Chem. Sci., 5(4): 1682-1687. DOI : http://dx.doi.org/ 10.4314/ijbcs.v5i4.30

Akpo Y, Kpodékon MT, Djago Y, Licois D, Youssao IAK. 2012. Vaccination of rabbits against coccidiosis using precocious lines of Eimeria magna and Eimeria media in Benin. Vet. Parasitol., 184: 73-76. DOI : http://dx.doi.org/ 10.1016/j.vetpar.2011.08.012

Andersen S, Mostue BA. 2011. Risk analysis and risk management approaches applied to the petroleum industry and their applicability to IO concepts. Saf. Sci., 50: 2010-2019. DOI : http://dx.doi.org/10.1016/j.ssci.2011.07. 016
Colombo T, Zago LG. 2003. Les Lapins (1 $1^{\text {ère }}$ édn). Les Presses de Grafiche: Milan.

Djago Y, Kpodékon M, Lebas F. 2010. Le Guide Pratique de l'Eleveur de Lapins sous les Tropiques (2 $2^{\mathrm{ème}}$ édn). Immediat' Presse: Cotonou.

Kpodékon M, Gnimadi A, Djago Y, Koutinhouin B, Farougou S. 2000. Rabbit production and network in Benin in 1998. 7th World Rabbit Congress, 103-110.

Pakandl M, Jelinkova A. 2006. The rabbit coccidium Eimeria piriformis: Selection of a precocious line and life-cycle study. Vet. Parasitol., 137: 351-357. DOI : http://dx.doi.org/10.1016/j.vetpar.2006. 01.012

Shivaramaiah C, Barta JR, Hernandez-Velasco X, Téllez G, Hargis BM. 2014. Coccidiosis: recent advancements in the immunobiology of Eimeria species, preventive measures, and the importance of vaccination as a control tool against these Apicomplexan parasites. Vet. Méd.: Res. and Rep., 5: 23-34. DOI: http://dx.doi.org/10. 2147/VMRR.S57839

Xu Q, Song Y, Xu L, Yan R, Skah MAA, LI X. 2008. Vaccination of chickens with a chimeric DNA vaccine encoding Eimeria tenella TA4 and chicken IL-2 induces protective immunity against coccidiosis. 2008. Vet. Parasitol., 156: 319-323. DOI : http://dx.doi.org/ 10.1016/j.vetpar.2008.05.025

SAS. 2010. SAS/STAT. User's Guide (6 ${ }^{\text {th }}$ edn). Inst. Inc.: New York. 